\title{
- a castax \\ Polymicrobial anaerobic infection with a deep abscess in the supraspinous fossa following a subacromial corticosteroid injection
}

\author{
John G Skedros, ${ }^{1}$ Marshall K Henrie, ${ }^{1}$ Ethan D Finlinson, ${ }^{1}$ Joel D Trachtenberg ${ }^{2}$
}

${ }^{1}$ Utah Orthopaedic Specialists, Murray, Utah, USA

Intermountain Medical Center, Murray, Utah, USA

Correspondence to Dr John G Skedros, jskedrosmd@uosmd.com

Accepted 5 November 2018

Check for updates

(c) BMJ Publishing Group Limited 2018. Re-use permitted under CC BY-NC. No commercial re-use. See rights and permissions. Published by BMJ.

To cite: Skedros JG, Henrie MK, Finlinson ED, et al. BMJ Case Rep

2018:11:e226598

doi:10.1136/bcr-2018-

226598

\section{SUMMARY}

In September 2015, a male aged 61 years with poorly controlled diabetes (his only medical problem) had left shoulder surgery that included an arthroscopic acromioplasty with debridement of suture material from a rotator cuff repair done 10 years prior. A subacromial corticosteroid injection was given 7 months later for pain and reduced motion. Three weeks later a fulminate infection was evident. Cultures grew Propionibacterium acnes. Treatment included two arthroscopic debridement surgeries and 8 weeks of intravenous antibiotics (primarily daptomycin). Eight weeks after the cessation of the antibiotics, purulence recurred and tissue cultures then grew Staphylococcus epidermidis. Several additional surgeries were needed to control the infection. We failed to recognise that an abscess that extended from the subacromial space across the entire supraspinous fossa. We report this case to alert clinicians that a seemingly innocuous subacromial corticosteroid injection can lead to an atypical infection and also extend into the supraspinous fossa.

\section{BACKGROUND}

A male patient with diabetes with poor glycaemic control had, during a 7-month period in 2016, multiple surgical irrigation and debridement (I and D) procedures for a recurrent deep infection of the left shoulder. The anatomic distribution of the infection was highly unusual because it had spread across the entire supraspinous fossa of the scapula. The initial infection caused by Propionibacterium acnes occurred 3 weeks after a subacromial corticosteroid injection was given for impingement symptoms. (In 2016, the taxonomic affiliation of $P$. acnes was changed to Cutibacterium acnes). ${ }^{1}$ After two surgical debridements and an 8-week course of intravenous and oral antibiotics, an infection recurred in the left shoulder. Cultures then grew Staphylococcus epidermidis. Eradication of the infection was difficult, requiring several additional $\mathrm{I}$ and D surgeries over a 5 -month period and an extended course of intravenous and oral antibiotics. Our literature search did not reveal any reports of $P$. acnes or S. epidermidis causing postinjection abscesses extending beyond the shoulder region such as was found in our patient. The unusual spread of the infection and the growth of these two microbes are the main novel aspects of this case. We also discuss this case in the perspective of a review of the literature of infections following subacromial injections and non-arthroplasty shoulder surgeries similar to those that were performed in our patient (arthroscopic acromioplasty with debridement and open rotator cuff tendon repair).

\section{CASE PRESENTATION}

A right-hand-dominant male patient aged 61 years with insulin-dependent diabetes (height $177.8 \mathrm{~cm}$; weight $104 \mathrm{~kg}$; body mass index 33.4) was referred by an infectious disease specialist (JDT) to our clinic for the surgical management of a recurrent deep infection of the left shoulder. The patient worked as a commercial property developer and had only recently started using insulin for diabetes. Poor glycaemic control was clear from his self-reported history and a high haemoglobin A1C (12.8\%; normal is $<5.7 \%$ ). He denied having any other medical problems and he did not drink alcoholic beverages or smoke. As detailed below, about 4 months prior the patient had two arthroscopic debridement procedures and completed an 8-week course of antibiotics for a $P$. acnes infection of his left shoulder region. When he was first seen in our clinic for the recurrent infection, 2 months had passed from the completion of the 8-week course of intravenous and oral antibiotics.

His left shoulder problems started approximately 11 years prior. At that time he was not diabetic and had a successful and uncomplicated open repair of a torn supraspinatus tendon. In September 2015, he had a second left shoulder surgery for impingement symptoms, and this included an arthroscopic acromioplasty with debridement of suture material from the prior cuff repair. Around that time, metformin was prescribed for new-onset diabetes, but he was not compliant with taking this medication. A subacromial corticosteroid injection was given 7 months (April 2016) later for persistent pain and progressively reduced motion. Three weeks after the injection, a large abscess had formed and it extended from the glenohumeral joint and subacromial space to the subcutaneous tissue over the acromion. His blood glucose was 340 (normal: 65-99). An aspiration of purulent fluid grew $P$. acnes in fortified thioglycolate broth culture in anaerobic conditions. In addition to two surgical debridement procedures, antibiotic treatment by his orthopaedic surgeon initially included a 10-day course of intravenous vancomycin and oral levofloxacin $(750 \mathrm{mg}$ 


\section{Inflammatory Markers}

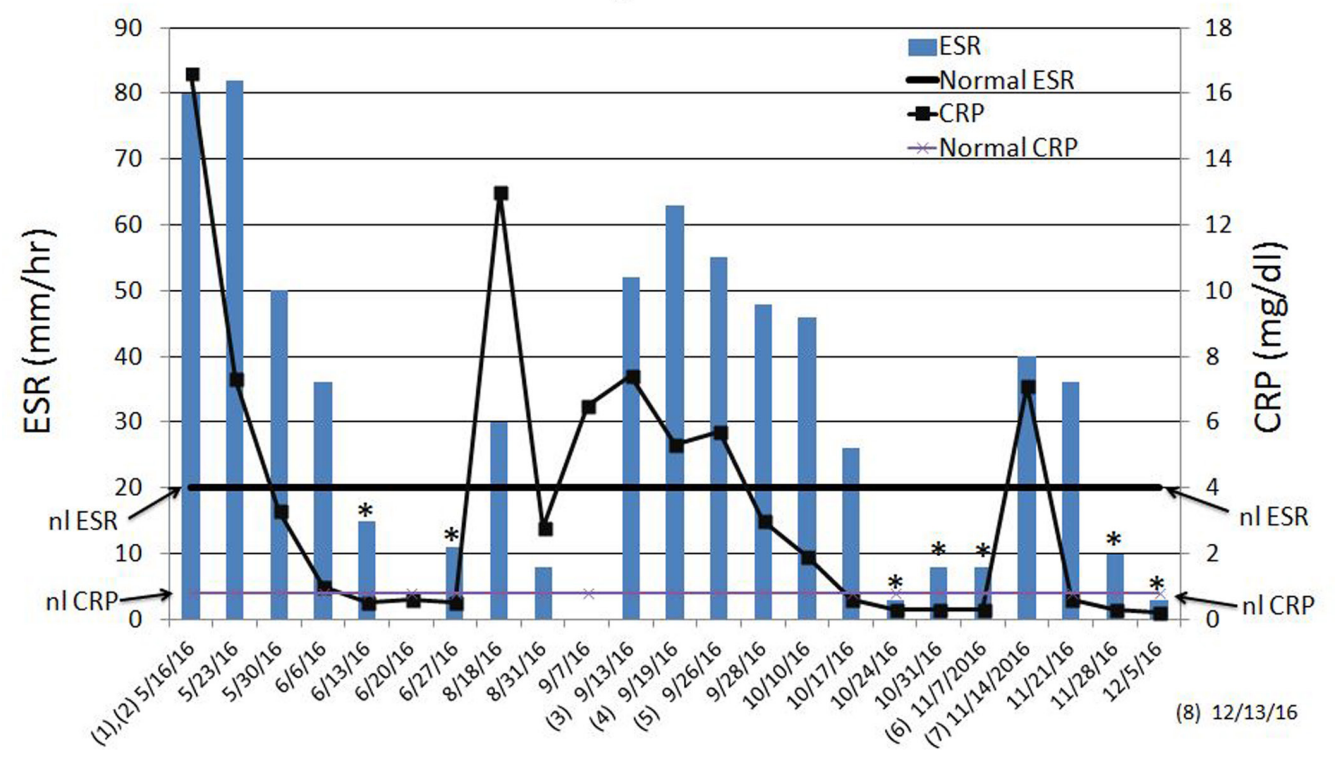

Figure 1 Erythrocyte sedimentation rate (ESR) and C reactive protein (CRP) values, and dates of surgical debridements (numbers 1-8). Asterisks indicate dates when both ESR and CRP were normal.

day). An infectious disease specialist (JDT) who was then consulted discontinued these antibiotics and started a 6-week course of intravenous daptomycin. Although penicillin is often the antibiotic treatment for this type of infection, it was not used because he was allergic to it.

\section{TREATMENT}

Approximately 8 weeks after completing this treatment the infection recurred, with pus draining through sinus tracts that had developed at incisions made during the prior arthroscopic I and D surgery. The patient was then referred to our clinic.

While in our care, the patient underwent several additional surgeries between 13 September 2016 and 13 December 2016 (figure 1). S. epidermidis grew from tissue and fluid cultures taken during from our first debridement surgery. The abscess extended from the subacromial region and glenohumeral joint into the overlying subcutaneous tissue through fenestra in the anterior deltoid insertion. In addition to purulent fluid, findings also included a $2.5 \mathrm{~cm}$ wide and $2.0 \mathrm{~cm}$ retracted supraspinatus tendon tear, a polymer suture anchor, remnants of braided sutures and very mild glenohumeral arthritis. There was no myonecrosis or necrotising fasciitis. Because of extensive inflammation and purulence, the deltoid was not repaired and the wound was left open. A sponge was placed and this extended from the superficial to deeper aspect of the wound, and a wound vacuum was applied (V.A.C.Ulta Negative Pressure Wound Therapy System, KCI Medical, USA). Antibiotic treatment included daily intravenous daptomycin and oral rifampin ( $300 \mathrm{mg}$ twice a day) in order to cover both $P$. acnes and S. epidermidis. ${ }^{2}$ The wound vacuum sponge was changed every 3 days.

Two additional debridement surgeries were done during the next 5 weeks. During that time the patient progressively improved and his left shoulder remained free of signs of infection. On 24 and 31 October 2016, the patient's erythrocyte sedimentation rate (ESR) and $\mathrm{C}$ reactive protein (CRP) levels were normal (figure 1). However, on 3 November 2016, a routine check-up revealed an area of swelling over the region of the left scapular spine that was not present on prior examinations. An aspiration with an 18-gauge needle yielded greenish-grey fluid. This did not grow any organisms in culture (the patient was still on intravenous daptomycin and oral rifampin). A magnetic resonance (MR) scan was then obtained (figure $2 \mathrm{~A}-\mathrm{L}$ ), which was the first one that had been obtained during the entire course of his infection treatments. The MR images showed an abscess that extended across the entire supraspinous fossa and there was a tract that connected the deeper portion of the abscess to the subcutaneous tissues in the mid-scapula region (figure $2 \mathrm{~F}-\mathrm{H}$ ).

To eradicate this residual locus of infection, two additional surgeries were done during a 1-month period. After the final surgery, the patient received intravenous daptomycin and oral rifampin for four more weeks. This treatment was then followed by a 3-month course of oral doxycycline hyclate $100 \mathrm{mg}$ twice a day and oral rifampin $300 \mathrm{mg}$ twice a day.

\section{OUTCOME AND FOLLOW-UP}

At 3 months after the final surgery, he reported having good shoulder function and he did not require pain medication. Physical examination showed $145^{\circ}$ of active flexion and $130^{\circ}$ of active abduction. At 15 months after his final surgery, he reported continuing good shoulder motion with only mild weakness, no significant pain with daily activities and remained free of infection.

\section{DISCUSSION}

Subacromial corticosteroid injections are commonly used to treat shoulder pain. ${ }^{3-5}$ Complications of this procedure are uncommon, and include bleeding, allergic reaction, tendon rupture, muscle necrosis and deep and superficial infections. ${ }^{35} 6$ A retrospective study by French rheumatologists ${ }^{7}$ reported an infection rate following glenohumeral corticosteroid injections of approximately 1 in 75000 . With the introduction of the corticosteroid being packaged in a sterile syringe, the frequency dropped to 1 in 162000 . The rate of infections following subacromial corticosteroid injections is not known, but is believed to be even less. ${ }^{8}$ The rarity of $P$. acnes infections and/ 

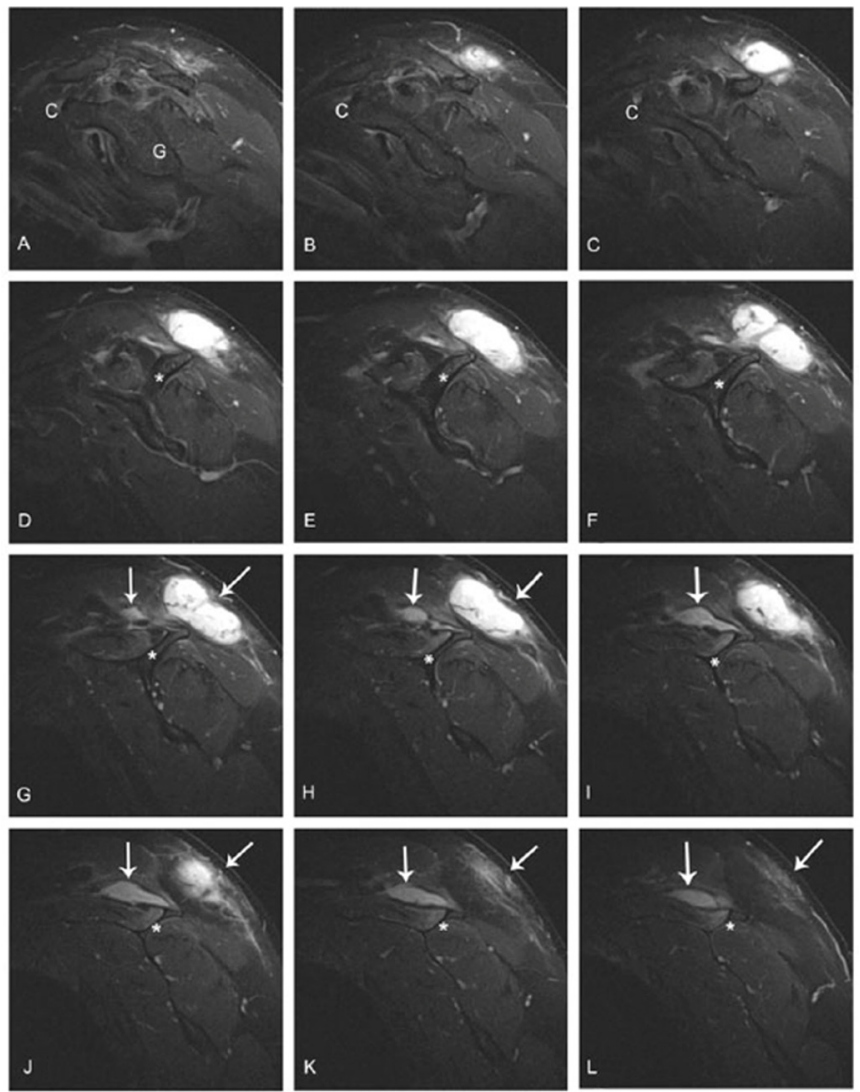

Figure 2 Sequential sagittal-plane MR images from lateral to medial (A to $L$, respectively) through the patient's left shoulder and scapula. A sinus-like tract between the deeper and superficial abscesses can be seen in images $F, G$ and $H$. At the left of centre in the first three images, the $\mathrm{C}=$ coracoid; $\mathrm{G}=$ glenoid; * =spine of scapula; vertical arrow=extension of infection along the region of the supraspinous fossa; angled arrow=infection in subcutaneous tissues. In images $D$, $E$ and $F$, there is a signal change in the scapular spine adjacent to the abscess. This could represent osteomyelitis that was not recognised until review of this case (discussed just prior to conclusion paragraph).

or S. epidermidis infections following subacromial corticosteroid injections is one of the two unusual aspects of our case report.

Although primarily recognised for its role in acne, P. acnes is an anaerobic Gram-positive bacterium that forms part of the normal flora of the $\operatorname{skin}^{9}{ }^{10}$ and has emerged as a common cause of infections after shoulder arthroplasty and non-arthroplasty surgeries. ${ }^{11-16}$ But infections after shoulder surgeries and subacromial corticosteroid injections reported $>15$ years ago were more often attributed to Staphylococcus aureus, S. epidermidis and other Staphylococcus or Streptococcus species. ${ }^{5} 8$ 17-19 During that era, P. acnes was often considered to be a contaminant. ${ }^{1320}$ Since then $P$. acnes has become recognised as an increasingly common postoperative and postinjection pathogen of the shoulder region. ${ }^{11-16}{ }^{21-23}$ For example, Athwal et al ${ }^{13}$ reported on 39 deep infections after conventional open or miniopen rotator cuff repairs in 4886 patients who had surgeries performed between 1975 and 2003. P. acnes grew in culture from $51 \%$ of the cases. Herrera et $a^{22}$ reported on a series of 360 patients (mean age $=55$ years; range $40-64$ years) who underwent mini-open rotator cuff repairs immediately after arthroscopic subacromial decompressions. P. acnes was present in six of seven patients $(86 \%)$ with infections. Other causes of bacterial infections after these types of non-arthroplasty procedures include $S$. aureus, S. epidermidis, Staphylococcus warneri, Staphylococcus haemolyticus, Staphylococcus saccharolyticus, Micrococcus luteus, Achromobacter denitrificans, Peptostreptococcus magnus, Bacillis species, Corynebacterium species, Streptococcus species and Pseudomonas species. ${ }^{8} 1213151622-24$

In cases that are more similar to our patient's case, Horneff et $a l^{16}$ prospectively collected data on all shoulder arthroscopies performed by one surgeon from 1 January 2009 to 1 April 2013. Cultures were obtained in all revision shoulder arthroscopy cases performed for pain, stiffness or weakness, but none of these cases had clear suspicion of infection. In order to determine the false-positive rate for bacteria growth, two tissue cultures were taken from 32 primary shoulder arthroscopy cases where there was no concern for infection. Results showed that of a total of 1591 shoulder arthroscopies, 68 (4\%) were revision procedures performed for pain, stiffness or weakness. A total of 20 revision arthroscopies (29\%) had positive culture findings, and $16(24 \%)$ were positive for $P$. acnes. In the control group, one patient $(3 \%)$ had $P$. acnes growth.

Schneeberger et $a l^{21}$ reported shoulder infections that occurred in patients who had corticosteroid injections but no prior shoulder surgeries. The number of injections ranged from four to eight, but the specific location injected was not reported. In tissue cultures, $P$. acnes was identified in five patients, coagulase-negative Staphylococcus species in two and S. saccharolyticus in one shoulder. Similar to our patient, one of their patients had a mixed infection with $P$. acnes and a coagulase-negative Staphylococcus species. Unlike our patient, none of their patients had more than one debridement surgery, none had unusual spread of the infection, none had purulence and all debridement surgeries were arthroscopic.

S. epidermidis is a Gram-positive, catalase-positive, coagulase-negative, facultative anaerobe that normally colonises various areas of the skin, but it can act as an opportunistic pathogen when it enters the bloodstream. ${ }^{1025}$ S. epidermidis has recently developed significant resistance to several antibiotics and has become one of the leading causes of hospital-acquired infections. ${ }^{26}$ P. acnes and S. epidermidis form biofilms that protect them against antibiotic treatment and the immune system. ${ }^{27} 28$ For this reason, antibiofilm antibiotics such as rifampin are often added to enhance the treatment of these organisms. ${ }^{28}{ }^{29}$ Highdose intravenous penicillin is usually the drug of choice for treating $P$. acnes infections and rifampin is often used in combination with this antibiotic or others (eg, vancomycin or daptomycin). ${ }^{263031}$

Polymicrobial infections following rotator cuff repairs are not unusual; for example, 6/39 (15\%) infections reported by Athwal et $a l^{13}$ were polymicrobial. It is also possible that our patient's initial infection was polymicrobial (P. acnes and S. epidermidis). But because $S$. epidermidis did not grow initially, it could have been a later contaminant ${ }^{32} 33$ grown in cultures from the third debridement surgery (which was the first surgery performed after the infection had recurred). $P$. acnes might have persisted at that time but just failed to grow by the 14th day that the cultures were held. Although growth of this organism usually requires 9-14 days, ${ }^{3435}$ growth can take longer in some cases and this depends on various factors including the presence of a polymicrobial infection, characteristics of the tissue or fluid tested and the culture media used. ${ }^{12} 3637$ By contrast, S. epidermidis usually grows within 4 days. ${ }^{12} 38$ There is also evidence that $P$. acnes growth can be inhibited by $S$. epidermidis in some environments, ${ }^{25} 39$ which could explain why $S$. epidermidis grew but $P$. acnes failed to grow from cultures obtained during the patient's later surgical debridements. 
It is possible that our patient had an indolent infection at the time of his subacromial corticosteroid injection. Indolent $P$. acnes infections can be the cause of persistent shoulder pain and reduced motion for many months, or even years, after shoulder surgery or corticosteroid injections, and can present without fever or other typical systematic manifestations of infection..$^{1316212340}$ For example, Khan et al ${ }^{12}$ reported nearly 20 months (range $\sim 2-134$ months) as the mean time to an infection diagnosis after arthroscopic and open Latarjet surgeries in their 21 patients with infections. Herrera et $a l^{22}$ reported a mean time to surgical debridement was nearly 6 months (range $\sim 3-9$ months) in their seven patients with $P$. acnes infections after mini-open rotator cuff repairs. $P$. acnes infections of the shoulder are especially difficult to diagnose because they are asymptomatic for prolonged periods of time and they typically have normal white blood cell count, ESR and $\mathrm{C}$ reactive protein. ${ }^{14} 41$ Our patient's infection occurred 21 days after his subacromial corticosteroid injection, which was given at approximately 7 months after his arthroscopic surgery. Hiemstra et $a l^{8}$ found that the mean time from injection to surgical debridement was, similar to our case, 21 days $(7,17$ and 30 days in their three patients) even though their organisms were not anaerobic. By contrast, Schneeberger et al ${ }^{15}$ noted that their seven patients with postinjection infections (five had $P$. acnes) had symptoms for 10-36 months prior to having surgery. But, unlike the infection in our patient, their patients had comparatively mild infections (eg, no purulence). These various studies show that $P$. acnes presentations following shoulder surgeries or corticosteroid injections can be highly variable in terms of characteristics of presentation, chronology and severity. Because our patient had diabetes, it might seem unwise to perform a corticosteroid injection in an area that might harbour a low-grade infection. An alternative treatment could have been a local anaesthetic injection with shoulder manipulation and/or physical therapy.

The severity of our patient's infection was likely a combination of several factors, including the reduced immunity that occurs with insulin-dependent diabetes ${ }^{42}$ and the inadequacy of the earlier surgeries to debride all locations of the infection. Additionally, corticosteroids injected into musculoskeletal regions of these patients can further impair immunity, which can cause a new infection or an indolent infection to flare. ${ }^{5}$

Our review of the literature suggests that wound vacuums (negative pressure wound therapy (NPWT)) are infrequently used in the surgical management of deep infections of the shoulder. ${ }^{43}$ In the Athwal et $a l^{13}$ cohort with 39 deep shoulder infections after open rotator cuff repairs, they reported that between the surgical debridements done on each patient the wound was left open and packed with sterile gauze in 18 shoulders and closed over a drain in 21. Antibiotic-containing cement beads were used in five cases. By contrast, we used NPWT because it seemed to us to be a reasonable way to help enhance the eradication of our patient's infection by consistently removing fluids. ${ }^{44}$ However, we have since become aware that the use of a wound vacuum in this setting can actually increase the amount of bacteria. For example, in a prospective pilot study of the treatment of chronic leg and foot infection in 13 patients, Goss et $a l^{45}$ treated all patients with sharp surgical debridement followed by NPWT. One group had conventional NPWT and the other had NPWT with instillation (NPWTi) of quarter strength bleach to the wound. The NPWTi group had a statistically significant reduction in bacteria burden, while wounds treated with NPWT had an increase in bacteria burden over the 7-day treatment period. Similar findings have been reported in their subsequent prospective randomised trial of 20 patients with chronic lower extremity wounds. ${ }^{46}$ Although we could not locate studies that have evaluated NPWT versus NPWTi in treating deep infections of the shoulder, we conclude that NPWTi would have likely have helped reduce the time to the closure of our patient's posterior scapula wound.

The second unusual aspect of our case is that the infection spread beyond the typical subacromial/subdeltoid space and glenohumeral joint where subacromial injections are usually confined. Consequently, MR imaging did not seem necessary during the initial phase of our treatment. A routine MR of his shoulder would have shown that the infection had extended into the supraspinous fossa of the scapula. ${ }^{3}{ }^{47}$ In the earlier treatment of our patient's infection, it would have been useful to perform imaging in addition to radiography, for example, MR, CT or ultrasonography. The advantages of each modality are well known and could have been helpful in developing the differential diagnosis for our patient including osteitis, tumour and other pathologies. ${ }^{48}$ Our literature search also did not reveal any reports of $P$. acnes or $S$. epidermidis causing postinjection abscesses extending beyond the shoulder region such as was found in our patient. Although Achermann et $a l^{49}$ reported a $P$. acnes abscess in the superficial tissues over the shoulder of a female aged 82 years after primary shoulder arthroplasty, the abscess did not extend to the supraspinous fossa or nearby deep tissue planes. Gruson et $a l^{5}$ provided MR images of a septic subacromial bursitis (S. aureus) in a female aged 25 years after she had a subacromial corticosteroid injection. The extent and location of their patient's infection resembled our case because it extended posterior and medially from the glenohumeral region into the infraspinatus muscle and infraspinous fossa of the scapula. Hiemstra et $a l^{8}$ reported the case of a male aged 34 years who had a Staphylococcus species infection after a subacromial corticosteroid injection, and MR imaging showed that the infection extended 'into the posterior rotator cuff muscles'. How far medially and superficially the infection extended was not reported (no MR images or detailed description provided). Lan et $a l^{50}$ reported the case of a female aged 56 years who had a subacromial/subdeltoid infection extending into all of the rotator cuff muscles, and the organism was a viridans-group Streptococcus. Unlike our case, this patient did not have a shoulder injection (the infection occurred spontaneously following a strain).

During an independent review of our case it was suggested we may have missed an indolent osteomyelitis on the distal aspect of scapular spine, which is seen as signal changes on three of the MRI images shown in figure 2 D-F. While this was not initially recognised. it could help explain the long duration of his treatment. No bone biopsy or nuclear medicine scan was performed

\section{Learning points}

- Propionibacterium acnes and Staphylococcus epidermidis both grew from cultures of infected tissues but at different times during his treatment.

- The infection extended into the supraspinous fossa, supraspinatus muscle and overlying subcutaneum.

- In cases of recurrent shoulder infections, it would be important to obtain an MR scan that includes the scapula in order to avoid missing extensive spread of the infection.

- Deep infections/abscesses caused by these anaerobic bacteria after subacromial corticosteroid injections might require many surgical debridements and an extended course of antibiotics to eradicate the infection.

- These patients can regain good shoulder function, as was the final outcome in our patient. 
to explore the idea of osteomyelitis being the nidus of the postinjection abscess and fistula formation.

Contributors The authors contributions are the following:. JGS: planning, conduct, reporting, conception and design, acquisition of data, analysis and interpretation of data. MKH: planning, conduct, reporting, conception and design, analysis and interpretation of data. EDF: planning, conduct, reporting, conception and design, analysis and interpretation of data. JDT: conduct, reporting, acquisition of data, analysis and interpretation of data.

Funding The authors have not declared a specific grant for this research from any funding agency in the public, commercial or not-for-profit sectors.

Competing interests None declared.

Patient consent Obtained.

Provenance and peer review Not commissioned; externally peer reviewed.

Open access This is an open access article distributed in accordance with the Creative Commons Attribution Non Commercial (CC BY-NC 4.0) license, which permits others to distribute, remix, adapt, build upon this work non-commercially, and license their derivative works on different terms, provided the original work is properly cited and the use is non-commercial. See: http://creativecommons.org/ licenses/by-nc/4.0/

\section{REFERENCES}

1 Scholz CF, Kilian M. The natural history of cutaneous propionibacteria, and reclassification of selected species within the genus Propionibacterium to the proposed novel genera Acidipropionibacterium gen. nov., Cutibacterium gen. nov. and Pseudopropionibacterium gen. nov. Int I Syst Evol Microbiol 2016;66:4422-32.

2 Jugun K, Vaudaux P, Garbino J, et al. The safety and efficacy of high-dose daptomycin combined with rifampicin for the treatment of Gram-positive osteoarticular infections. Int Orthop 2013;37:1375-80.

3 Teoh KH, Jones SA, Gurunaidu S, et al. Methicillin-resistant Staphylococcus aureus infection of the subacromial bursa: an unusual complication following subacromial corticosteroid injection (a report of two cases). Shoulder Elbow 2015;7:182-6.

4 Blair B, Rokito AS, Cuomo F, et al. Efficacy of injections of corticosteroids for subacromial impingement syndrome. J Bone Joint Surg Am 1996:78:1685-9.

5 Gruson KI, Ruchelsman DE, Zuckerman JD. Subacromial corticosteroid injections. J Shoulder Elbow Surg 2008;17:\$118-30.

6 Yu CM, Chen CH, Liu HT, et al. Subacromial injections of corticosteroids and xylocaine for painful subacromial impingement syndrome. Chang Gung Med J 2006;29:474-9.

7 Seror P, Pluvinage P, d'Andre FL, et al. Frequency of sepsis after local corticosteroid injection (an inquiry on 1160000 injections in rheumatological private practice in France). Rheumatology 1999:38:1272-4.

8 Hiemstra LA, Macdonald PB, Froese W. Subacromial infection following corticosteroid injection. J Shoulder Elbow Surg 2003;12:91-3.

9 Chuang MJ, Jancosko JJ, Mendoza V, et al. The incidence of Propionibacterium acnes in shoulder arthroscopy. Arthroscopy 2015;31:1702-7.

10 Patel A, Calfee RP, Plante M, et al. Propionibacterium acnes colonization of the human shoulder. J Shoulder Elbow Surg 2009;18:897-902.

11 Perry A, Lambert P. Propionibacterium acnes: infection beyond the skin. Expert Rev Anti Infect Ther 2011;9:1149-56.

12 Khan U, Torrance E, Townsend R, et al. Low-grade infections in nonarthroplasty shoulder surgery. J Shoulder Elbow Surg 2017;26:1553-61.

13 Athwal GS, Sperling JW, Rispoli DM, et al. Deep infection after rotator cuff repair. J Shoulder Elbow Surg 2007;16:306-11.

14 Saper D, Capiro N, Ma R, et al. Management of Propionibacterium acnes infection after shoulder surgery. Curr Rev Musculoskelet Med 2015;8:67-74.

15 Schneeberger AG, Gilbart MK, Sheikh R, et al. Non-purulent low-grade infection as cause of pain following shoulder surgery: preliminary results. Chir Organi Mov 2009;93(Suppl 1):71-7.

16 Horneff JG, Hsu JE, Voleti PB, et al. Propionibacterium acnes infection in shoulder arthroscopy patients with postoperative pain. J Shoulder Elbow Surg 2015:24:838-43.

17 Small NC. Complications in arthroscopic surgery performed by experienced arthroscopists. Arthroscopy 1988;4:215-21.

18 D'Angelo GL, Ogilvie-Harris DJ. Septic arthritis following arthroscopy, with cost/benefit analysis of antibiotic prophylaxis. Arthroscopy 1988;4:10-14.

19 Randelli P, Castagna A, Cabitza F, et al. Infectious and thromboembolic complications of arthroscopic shoulder surgery. I Shoulder Elbow Surg 2010;19:97-101.

20 Settecerri JJ, Pitner MA, Rock MG, et al. Infection after rotator cuff repair. J Shoulder Elbow Surg 1999;8:1-5.

21 Schneeberger AG, Yian E, Steens W. Injection-induced low-grade infection of the shoulder joint: preliminary results. Arch Orthop Trauma Surg 2012;132:1387-92.
22 Herrera MF, Bauer G, Reynolds F, et al. Infection after mini-open rotator cuff repair. J Shoulder Elbow Surg 2002;11:605-8.

23 Kadler BK, Mehta SS, Funk L. Propionibacterium acnes infection after shoulder surgery. Int I Shoulder Surg 2015;9:139-44.

24 Mirzayan R, Itamura JM, Vangsness $\mathrm{CT}$, et al. Management of chronic deep infection following rotator cuff repair. J Bone Joint Surg Am 2000;82-A:1115-21.

25 Christensen GJ, Scholz CF, Enghild J, et al. Antagonism between Staphylococcus epidermidis and Propionibacterium acnes and its genomic basis. BMC Genomics 2016;17:152.

26 Leite B, Gomes F, Teixeira P, et al. In vitro activity of daptomycin, linezolid and rifampicin on Staphylococcus epidermidis biofilms. Curr Microbiol 2011;63:313-7.

27 Vuong C, Otto M. Staphylococcus epidermidis infections. Microbes Infect 2002; $4: 481-9$

28 Aubin GG, Portillo ME, Trampuz A, et al. Propionibacterium acnes, an emerging pathogen: from acne to implant-infections, from phylotype to resistance. Med Mal Infect 2014;44:241-50.

29 Furustrand Tafin U, Aubin GG, Eich G, et al. Occurrence and new mutations involved in rifampicin-resistant Propionibacterium acnes strains isolated from biofilm or devicerelated infections. Anaerobe 2015:34:116-9.

30 Crane JK, Hohman DW, Nodzo SR, et al. Antimicrobial susceptibility of Propionibacterium acnes isolates from shoulder surgery. Antimicrob Agents Chemother 2013;57:3424-6.

31 Rupp ME, Fey PD. Staphylococcus epidermidis and other coagulase-negative Staphylococci. In: Elsevier, editor. Mandell, Douglas, and Bennett's principles and practice of infectious diseases. 8 edn. Philadelphia: Elsevier Inc, 2015:2272-82.

32 Widerström M. Significance of Staphylococcus epidermidis in Health Care-Associated Infections, from Contaminant to Clinically Relevant Pathogen: This Is a Wake-Up Call!. J Clin Microbiol 2016;54:1679-81.

33 Hudek R, Sommer F, Kerwat M, et al. Propionibacterium acnes in shoulder surgery: true infection, contamination, or commensal of the deep tissue? J Shoulder Elbow Surg 2014;23:1763-71.

34 Schäfer P, Fink B, Sandow D, et al. Prolonged bacterial culture to identify late periprosthetic joint infection: a promising strategy. Clin Infect Dis 2008:47:1403-9.

35 Dodson CC, Craig EV, Cordasco FA, et al. Propionibacterium acnes infection after shoulder arthroplasty: a diagnostic challenge. J Shoulder Elbow Surg 2010;19:303-7.

36 Butler-Wu SM, Burns EM, Pottinger PS, et al. Optimization of periprosthetic culture for diagnosis of Propionibacterium acnes prosthetic joint infection. J Clin Microbiol 2011;49:2490-5.

37 Bossard DA, Ledergerber B, Zingg PO, et al. Optimal length of cultivation time for isolation of propionibacterium acnes in suspected bone and joint infections is more than 7 days. J Clin Microbiol 2016;54:3043-9.

38 Jeong J, Chang CL, Park TS, et al. Early Screening of oxacillin-resistant Staphylococcus aureus and Staphylococcus epidermidis from blood culture. J Korean Med SCi 2002:17:168-72.

39 Wang Y, Kao MS, Yu J, et al. A precision microbiome approach using sucrose for selective augmentation of staphylococcus epidermidis fermentation against propionibacterium acnes. Int J Mol Sci 2016;17:1870.

40 Millett PJ, Yen YM, Price CS, et al. Propionibacterium acnes infection as an occult cause of postoperative shoulder pain: a case series. Clin Orthop Relat Res 2011:469:2824-30.

41 Kanafani ZA, Corey GR. Daptomycin: a rapidly bactericidal lipopeptide for the treatment of Gram-positive infections. Expert Rev Anti Infect Ther 2007;5:177-84.

42 Chen AL, Shapiro JA, Ahn AK, et al. Rotator cuff repair in patients with type I diabetes mellitus. I Shoulder Elbow Surg 2003;12:416-21.

43 Jung $\mathrm{HJ}$, Song JH, Kekatpure AL, et al. The use of continuous negative pressure after open debridement for septic arthritis of the shoulder. Bone Joint J 2016;98-B:660-5.

44 Health Quality Ontario. Negative pressure wound therapy: an evidence-based analysis, Ont Health Technol Assess Ser 2006;6:1-38.

45 Goss SG, Schwartz JA, Facchin F, et al. Negative Pressure Wound Therapy With Instillation (NPWTi) better reduces post-debridement bioburden in chronically infected lower extremity wounds than NPWT alone. J Am Coll Clin Wound Spec 2012;4:74-80.

46 Yang C, Goss SG, Alcantara S, et al. Effect of negative pressure wound therapy with instillation on bioburden in chronically infected wounds. Wounds 2017;29:240-6.

47 Rhee RB, Chan KK, Lieu JG, et al. MR and CT arthrography of the shoulder. Semin Musculoskelet Radiol 2012;16:3-14.

48 Barile A, Bruno F, Mariani $\mathrm{S}$, et al. What can be seen after rotator cuff repair: a brief review of diagnostic imaging findings. Musculoskelet Surg 2017;101:3-14.

49 Achermann Y, Goldstein EJ, Coenye T, et al. Propionibacterium acnes: from commensal to opportunistic biofilm-associated implant pathogen. Clin Microbiol Rev 2014;27:419-40.

50 Lan S-M, Lin C-L, Wang C-K, et al. Synchronous subacromial and subdeltoid bursal abscess and pyomyositis of rotator cuff muscles caused by viridans streptococcus. Shoulder Elbow 2012;4:196-8. 


\section{Unusual association of diseases/symptoms}

Copyright 2018 BMJ Publishing Group. All rights reserved. For permission to reuse any of this content visit https://www.bmj.com/company/products-services/rights-and-licensing/permissions/

BMJ Case Report Fellows may re-use this article for personal use and teaching without any further permission.

Become a Fellow of BMJ Case Reports today and you can:

- Submit as many cases as you like

- Enjoy fast sympathetic peer review and rapid publication of accepted articles

Access all the published articles

- Re-use any of the published material for personal use and teaching without further permission

For information on Institutional Fellowships contact consortiasales@bmjgroup.com

Visit casereports.bmj.com for more articles like this and to become a Fellow 